\title{
External Anatomical Shapes Reconstruction from Turntable Image Sequences using a Single off-the-shelf Camera
}

\author{
Teresa C. S. Azevedo ${ }^{*}$, João Manuel R. S. Tavares ${ }^{+}$and Mário A. P. Vaz \\ * INEGI - Inst. de Eng. Mecânica e Gestão Industrial \\ LOME - Lab. de Óptica e Mecânica Experimental \\ FEUP - Faculdade de Engenharia da Universidade do Porto, PORTUGAL \\ + INEGI, LOME \\ DEMEGI - Departamento de Engenharia Mecânica e Gestão Industrial, FEUP, PORTUGAL
}

Received $10^{\text {th }}$ November 2007; revised $3^{\text {rd }}$ May 2008; accepted $11^{\text {th }}$ June 2008

\begin{abstract}
Three-dimensional (3D) human body reconstruction and modeling from two-dimensional (2D) images is a topic of great interest and research but still remains a difficult problem to solve. Volumetric based methods are commonly used to solve this type of problem. With those methods, from a turntable image sequence of an object, a 3D model can be built. The final goal of the work presented in this paper is to reconstruct all-round 3D models of external anatomical shapes with good accuracy and photorealistic appearance, using a single off-theshelf camera and a volumetric method.
\end{abstract}

Key Words: Computer Vision, 3D Reconstruction, Volumetric methods, Camera calibration.

\section{Anatomical shapes 3D reconstruction}

3D imaging of real objects has become a strong research topic in Computer Vision, mostly due to the increasing improvements done in different computational resources. Particularly, 3D reconstruction of anatomical shapes promises to open a very wide variety of possible applications like in medicine, virtual reality and industry, among many others.

3D geometric reconstructions of the human body were initially developed for ergonomic purposes in the automotive and aeronautics industry [37]. In those days, the used models consisted of a simple articulated skeleton to define human posture, with the body being represented by simple geometric primitives, such as cylinders or parallelepipeds [7]. Currently, 3D models of anatomical shapes are much more realistic and are usually built using contact, range- or image-based methods.

A briefly review on several reconstruction methods of anatomical shapes is given in the next sub sections. Then, in section 2, are pointed out usual classification criteria for some camera calibration methods, indispensable for accurate 3D reconstructions. Afterwards, in section 3, a succinct explanation of the

Correspondence to: <teresa.azevedo@fe.up.pt>

Recommended for acceptance by João Manuel R. S. Tavares and Renato Natal Jorge ELCVIA ISSN: 1577-5097

Published by Computer Vision Center / Universitat Autonoma de Barcelona, Barcelona, Spain 
methodology followed in this work is given. The conducted experiments, results and their analysis are presented in section 4. Finally, section 5 presents the main conclusions and future directions of our work.

\subsection{Contact methods}

Contact methods mean the use of some kind of devices that are in touch with zones of the human body. Main disadvantage of these methods is that, as the probe device must touch the skin, some deviations in the results could appear, because of the referred mechanical contact. Moreover, the data acquisition can be very time consuming and biologically unsafe; for example, a contact with damaged skin can cause an infection [18]. Nowadays, in the class of contact methods for human body 3D reconstruction, the use of fluids, such as water, has been considered a better alternative to the employment of mechanical devices [11].

\subsection{Range-based methods}

A range image is a collection of distance measurements from a well known 3D reference coordinate system to surface points on the object to be reconstructed. Usually, high quality 3D models of a static human body are obtained using commercial scanners based on this principle. Generally, these devices are expensive but easy to handle and the $3 \mathrm{D}$ reconstruction process is considerable fast [30].

Laser scanning, (e.g. [20, 41, 45]) and structured light pattern methods, (e.g. [5, 40, 49]) are the rangebased technologies most widely used to build 3D human models. Main contribution factors of these methods are their precision and simplicity of use. Another factor is the wide range of software packages available to process and edit the acquired data in order to build a 3D model for the object involved and then to determine characteristic measures on the same.

These methods are generally based on the triangulation principle [14] and can provide millions of points, often supplied with the related colour information as well. Acquisition time depends on the size of the object to be reconstructed and on the technology employed. However, these methods usually require for the object to remain stationary during the acquisition process. Thus, it is difficult to obtain stable reconstruction results of the human body, as humans always move, even slightly, during the data acquisition [26]. Other common drawbacks of these systems are the possibility of missing data points; namely, where the laser or projected pattern is occluded, and erroneous reconstruction data due to specularities of the objects surfaces [9].

\subsection{Image-based methods}

Other usual 3D static human reconstruction methods are image-based. They are an alternative and less expensive solution based on image measurements. Images to be used in the $3 \mathrm{D}$ reconstruction process can be acquired from video sequences or from photo-cameras.

\subsubsection{Multi-view geometry}

Multi-view geometry consists on reconstructing the 3D shape of an object from correspondences established along the image sequences acquired from different viewpoints, (Figure 1).

Multi-view geometry is considered an evolution of stereo-based methods, where only two images are used: first, correspondences between points in both images are established (matched) and then their position in 3D space is determined by triangulation [15]. Unfortunately, the first matching step is far from having an easy solution. Firstly, if the object to reconstruct has a smooth surface and low or constant texture, feature extraction may be incorrect, or even impossible to be established, since the local appearance is the same for every neighbourhood of the possible features to be matched. Secondly, matching correspondence cannot be established by just comparing local image measurements unless the object has a Lambertian surface; that is, its appearance does not change significantly along the consecutive viewpoints considered. To overcome these difficulties, variations and improvements to the original method appeared. The most common is the use 
of stereo-photogrammetry, in which the human body is "marked" with artificial fiducials (e.g. points) (e.g. $[6,33])$.

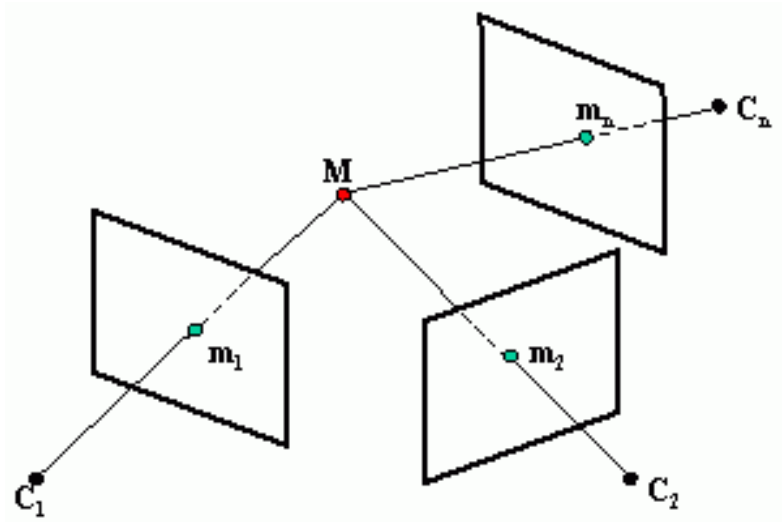

Figure 1: Given the correspondences between pixels $m_{1}, m_{2}$ and $m_{n}$, i.e., the projections of the same $3 \mathrm{D}$ point $\mathbf{M}$, the $3 \mathrm{D}$ coordinates of this point can be obtained by intersecting the optical rays that pass through the camera centers $C_{1}, C_{2}$ and $C_{n}$ and respective image points $m_{1}, m_{2}$ and $m_{n}$

\subsubsection{Volumetric methods}

Previous image-based methods often fail to reconstruct objects with complicated shapes, like the human body, essentially because of its high level of smoothness [47]. Thus, volumetric methods appeared recently as a versatile alternative for object $3 \mathrm{D}$ reconstruction, mainly for smooth objects [21].

These new methods are also image-based and are characterized by the use of a volumetric representation to describe the objects to be reconstructed [27]. Thus, the final reconstructed model is an aggregation of elementary volumetric primitives (e.g. voxels), representing a volume in the 3D space, where each elementary volume has the corresponding photogrammetric properties of the real object involved.

The first volumetric methods proposed were based on the visual hull concept [23] and denominated as shape-from-silhouettes methods. They build 3D models of the objects through the interception of conical volumes defined by the objects silhouettes in the acquired images and the associated image projection centres (Figure 2). Silhouette images can be easily extracted if the images are acquired in a controlled background setup. Models accuracy depends on the number of views used, the positions/orientations of each viewpoint, the calibration quality and the objects complexity. Unfortunately, these methods cannot deal with objects concavities and an accurate camera calibration procedure is required.

Some work examples for 3D human body reconstruction using image silhouettes can be found, for instance, in $[10,36,44]$.

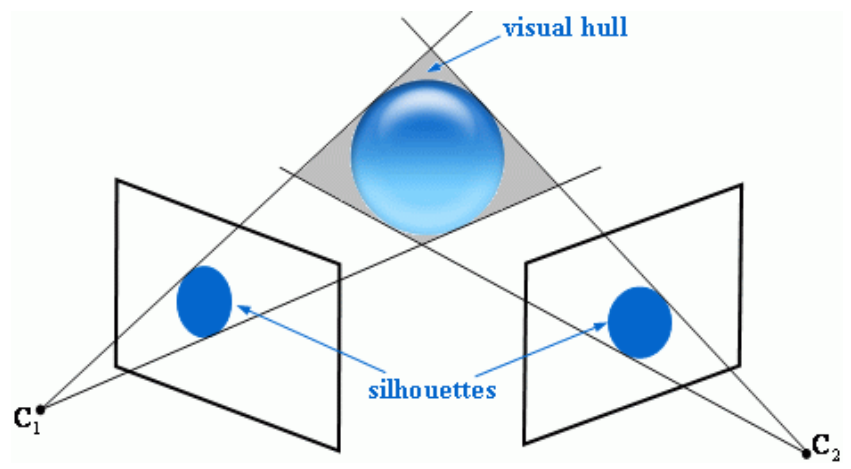

Figure 2: Visual hull of an object obtained from two viewpoints $C_{1}$ and $C_{2}$ 
Volumetric methods evolved in a way to test if each voxel belongs to the object of interest by using photo-consistency criteria. As an example, we can find the Space Carving reconstruction method presented in [22]. With this method, the resulting 3D model is based on photo hull that it is defined as the largest volume of voxels that are photo-consistent along all viewpoints considered (Figure 3). Voxels photoconsistency is checked statistically: a voxel is considered consistent if the mean deviation of the pixels color, which results from the voxel image projection, is below a predefined threshold level. One problem with this method is the determination of that threshold value, which is often obtained through experimentation until reasonable results are obtained. Usually, a low threshold level is suitable for areas with low texture, while highly textured areas or with sharp edges need higher threshold values.

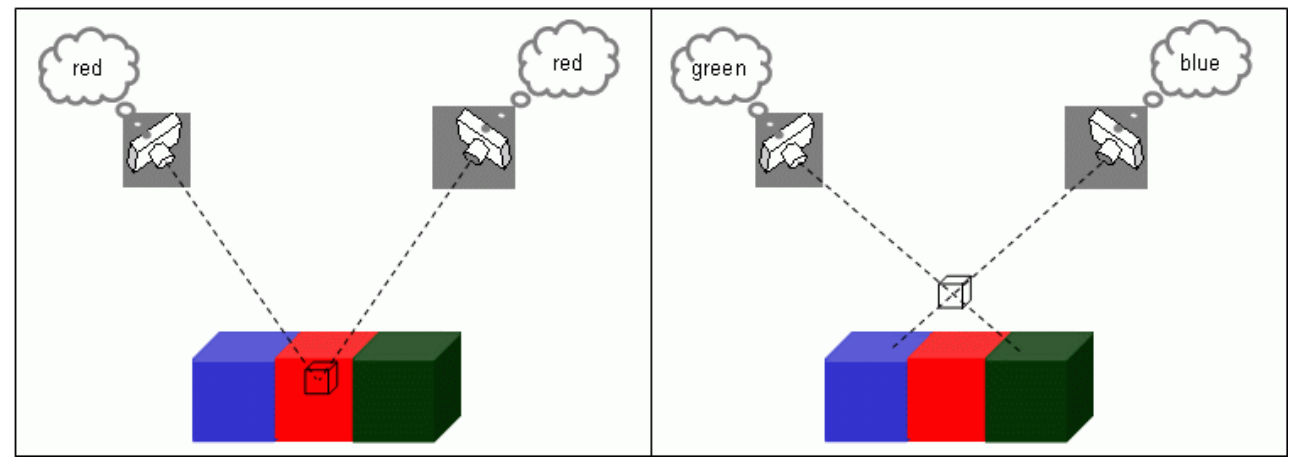

Figure 3: If a voxel belongs to the object surface, it will reproject the same color on all viewpoints were it is visible (left); however, if the projections of a voxel have different colors in some of the viewpoints, it is photo-inconsistent and, consequently, should not belong to the object surface (right)

In this work we followed the Generalized Voxel Coloring (GVC) method [34], which was derived from the Voxel Coloring method proposed in [32]. GVC allows configurations in which the camera used surrounds the objects completely, so all of the objects surfaces are visible in at least one image and therefore can be totally reconstructed. In GVC, voxels colour-consistency is checked over the entire set of input images on which they are visible, so the 3D models built can be more accurate, more realistic and better projected according to new viewpoints.

From a reconstructed volumetric model, a polygonal surface approximation can be built using, for example, the Marching Cubes algorithm [25]. Basically, this algorithm extracts a polygonal surface from the volumetrical data. Thus, it proceeds through the voxelized model, and, for each voxel, it determines the polygon(s) needed to represent the patch of the isosurface that passes through the referred voxel.

\subsubsection{Model-based methods}

Model-based approaches are now very common, in particular for monocular image sequences or rangebased methods. For example, the use of these model-based approaches is widespread in graphic communities, especially for animation purposes, where the goal is to build dynamic 3D models or for tracking a moving person. Generically, model-based methods use a prior knowledge of the objects geometry in order to constrain the shapes recovery process in the presence of visual ambiguities. Thus, they can reduce the influence of noisy, sparse or outlier data in shape $3 \mathrm{D}$ reconstruction, providing therefore more accurate 3D models [28].

For human 3D reconstruction, these approaches use a pre-defined human model or training data as reference to constraint or guide the interpretation of the image data acquired. Such models should support for a broad variety of possible poses, while being adaptable to different human shapes. At the same time, the set of parameters to describe the models pose should be kept as small as possible, because each additional parameter increases the dimensionality of the problem involved [19]. 


\section{Camera calibration methods}

Camera calibration tries to find the map between the 3D world and the $2 \mathrm{D}$ image plane. Assuming a perspective projection model, camera calibration means the determination of intrinsic and extrinsic camera parameters. Usually, calibration methods that require a calibration pattern, a special object with well known 3D world coordinates, are applied off-line. They can be classified according to a number of criteria [12]:

- Linear versus nonlinear:

- linear methods (e.g. [1, 17]): they use linear equation systems to determine the camera parameters; although fast and easy to implement, they are not suitable for applications where good accuracy is required or when the lens distortion needs to be modeled;

- non-linear methods (e.g. $[8,16,39,48])$ : the camera parameters are obtained through iteration methods; although slower than the previous ones, they can calibrate nearly any model and the accuracy usually increases by increasing the number of iterations; these methods require a good initial estimate, commonly obtained using linear methods, in order to guarantee the numerical convergence.

- Implicit versus explicit:

- implicit methods (e.g. [3, 8, 48]): the camera is calibrated without explicitly computing its physical parameters; although the results can be used for 3D measurement and generation of image coordinates, they are useless for camera modeling as the exact values of some camera parameters are not estimated;

- explicit methods (e.g. $[4,16,39])$ : all physical camera parameters are obtained.

- One single optimization versus multistep:

- one single step (e.g. [3, 8]): for each cycle of the resolution process, all camera parameters are updated;

- multistep (e.g. [4, 16, 39, 43]): in each step, a distinct subset of the camera parameters are obtained, using the initial estimates for those parameters already determined; good initial estimates can be quickly determined assuming some simplifications on the camera model, which will be progressively improved in the next optimization step.

- Planar versus non-planar:

- planar (e.g. $[4,39])$ : when all the 3D points of a calibration pattern belong to same plane; thus, the inaccuracy of the points coordinates is reduced, since one of them is null;

- non-planar: within this group, are the methods that require to know the relation between the different planes (usually, they choose to use a dihedron, that is, when the planes make an angle of $90^{\circ}$ between each other - e.g. $\left.[3,8,16,39]\right)$ and the methods that do not need to know the relation between the planes positions (usually, they can be image acquisitions of the same planar pattern in different space positions - e.g. [46, 48]).

\section{Used methodology}

Figure 4 summarizes the methodology followed in this work for the 3D reconstruction of anatomical shapes from turntable image sequences using a single off-the-shelf camera. First, for each object to be reconstructed, two image sequences are acquired:

- a first one, by moving a planar chessboard calibration pattern freely in 3D space; 
- and a second sequence, with the object to be reconstructed placed on a simple turntable device, with the same chessboard pattern beneath it; keeping the camera untouched, the second sequence of images is acquired, by spinning the turntable device until a full rotation is performed.

No restrictions are made on the number of images to acquire, neither is it necessary to know the rotation angle between two consecutive images of the second image sequence.

Even when the scene background has low colour variation, the photo-consistency criterion considered may not be sufficient for correct 3D reconstructions [31]. Also, since the used calibration pattern rotates together with the object to be reconstructed, it will not be considered has background and, consequently, will be reconstructed as if it belongs to the object. Thus, on the second image sequence, the segmentation between background and object is performed. For that, we use some basic image processing algorithms: a Sobel operator is used to detect the object contours; these are then dilated and the interest regions are filled; finally, erosion and opening are applied, in order to remove false regions and to smooth the silhouettes found.

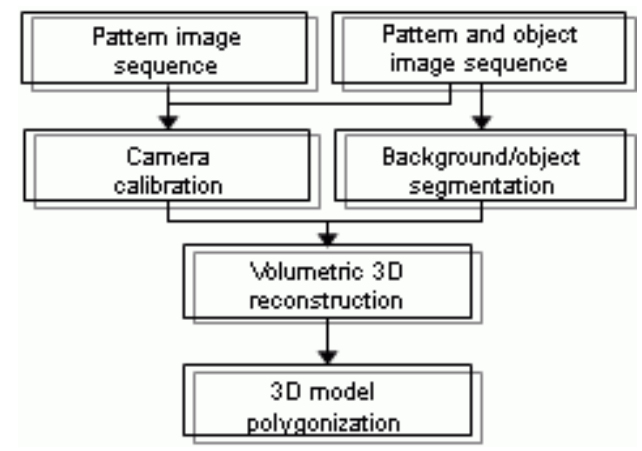

Figure 4: Methodology followed in this work to obtain the 3D model of objects from image sequences

The camera was calibrated using Zhang's calibration algorithm, [48]. Thus, intrinsic parameters (focal length and principal point) and distortion parameters (radial and tangential) were obtained from the first sequence. Then, using the second sequence, the extrinsic parameters (rotation and translation) were determined, for each viewpoint to be used in the reconstruction process.

Using the second image sequence and associated silhouette images, combined with the parameters determined by the calibration procedure used, the objects models are built using the GVC method implemented in [24].

Finally, the volumetric models are polygonized (smoothed) using the Marching Cubes algorithm [25].

\section{Experimental results}

This method was tested on three objects: a wooden doll (Figure 5), a human hand model (Figure 6) and a human torso model (Figure 7). This allowed testing of the methodology on objects considerably different in size and shape. For the doll and torso objects, a chessboard pattern with $10 \times 15$ squares was used, each measuring $24 \mathrm{~mm}$ by side. For the hand model object, the chessboard pattern had $6 \times 8$ squares, each measuring $30 \mathrm{~mm}$ by side. By using different calibration patterns, their influence on the accuracy of the calibration results was tested and, consequently, on the 3D reconstruction of the considered objects. All images were acquired with an off-the-shelf CCD camera.

\subsection{Calibration results}

The accuracy of the calibration results obtained was analyzed in two ways: by measuring the reprojection error of the calibration pattern points into all images of the second image sequences (Table 1) and by 
building a 3D graphical representation of the obtained camera extrinsic parameters (Figure 8). In these results, it can be noticed that the number of squares of the calibration patterns used strongly influences the accuracy of the calibration results obtained.

\subsection{Segmentation results}

The efficacy of the segmentation method used enabled to obtain good silhouette images for all considered objects (Figure 9). This is of high importance, since the silhouette information is used on the first test to define if a certain voxel belongs or not to the surface of the object under reconstruction. Only after a voxel has passed this test can the photo-consistency criterion be applied.

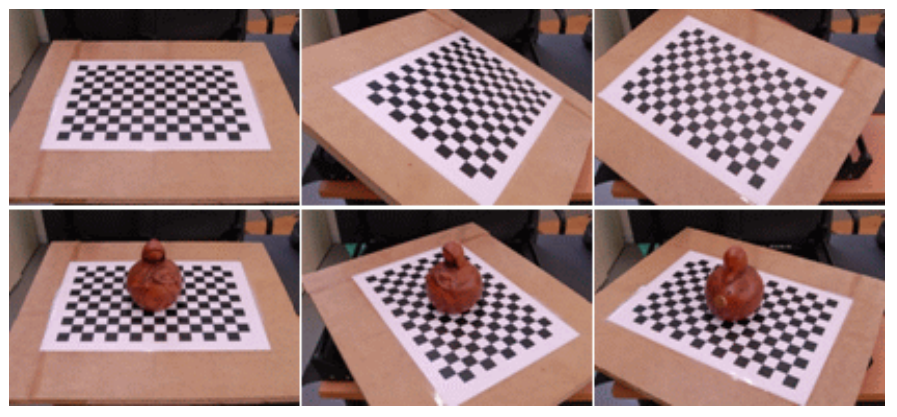

Figure 5: Three images of the first image sequence (top) and second image sequence (bottom) for the doll case

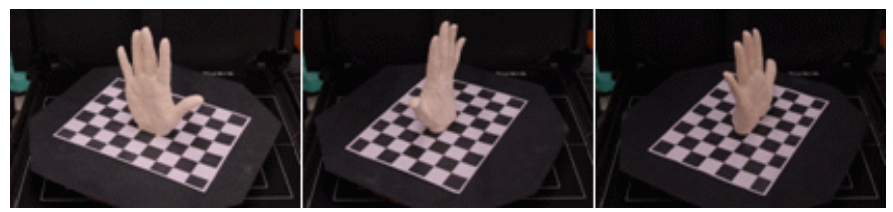

Figure 6: Three images of the second image sequence for the parallelepiped case

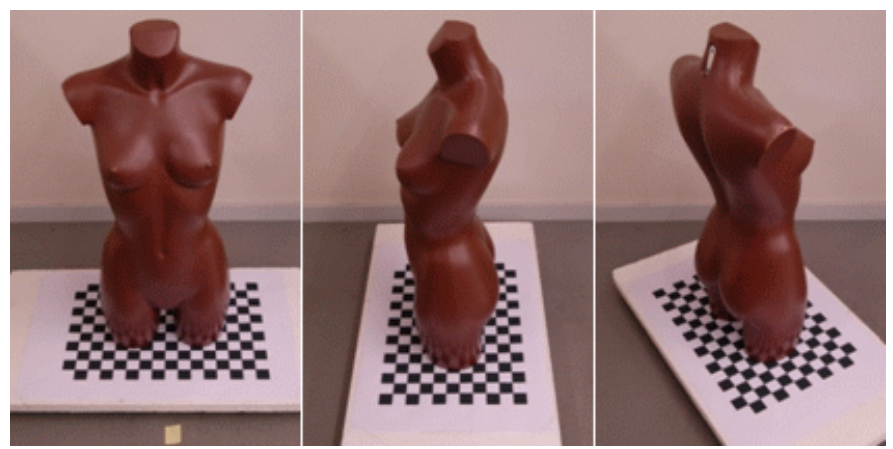

Figure 7: Three images of the second image sequence for the torso case

\begin{tabular}{|c|c|c|}
\hline \multirow{2}{*}{ Object } & \multicolumn{2}{|c|}{$\begin{array}{c}\text { Standard deviation of the } \\
\text { reprojection errors } \\
\text { (in pixels) }\end{array}$} \\
\hline & $x$ & $y$ \\
\hline doll & 0.21 & 0.63 \\
\hline hand model & 0.43 & 0.87 \\
\hline torso model & 0.16 & 0.61 \\
\hline
\end{tabular}


Table 1: Errors of the reprojection of the pattern points into all images of the second image sequences

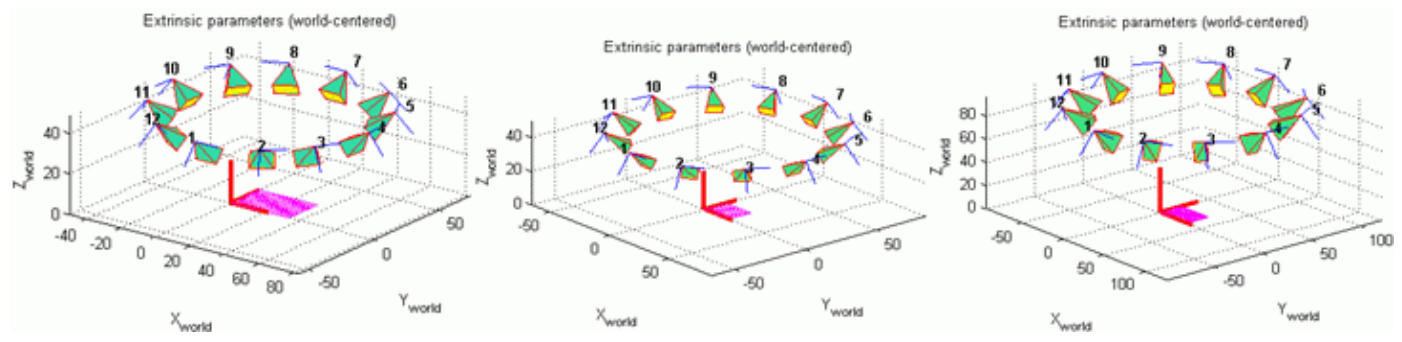

Figure 8: From left to right, graphical representation of the camera extrinsic parameters for the doll, hand model and torso model objects
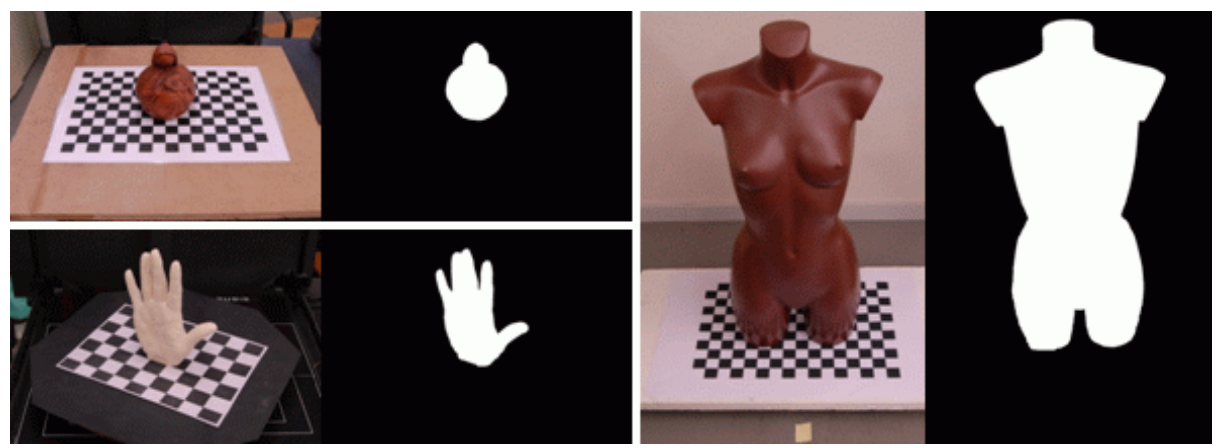

Figure 9: One result, per object, of the silhouettes segmentation step: on the left, the original images; on the right, the obtained segmented images

\subsection{Carving results}

With the second images sequences and respective silhouette images, combined with the camera calibration parameters, all objects models were successfully built, polygonized and smoothed, as it can be seen in Figure 10, Figure 11 and Figure 12. As noticed in Figure 12, the torso object was not completely successfully reconstructed. One possible reason for this may is the reflection of the calibration pattern on the surface of the torso. As consequence, the inferior volume of its reconstructed 3D model is not very accurate, both in terms of shape and colour (Figure 12).

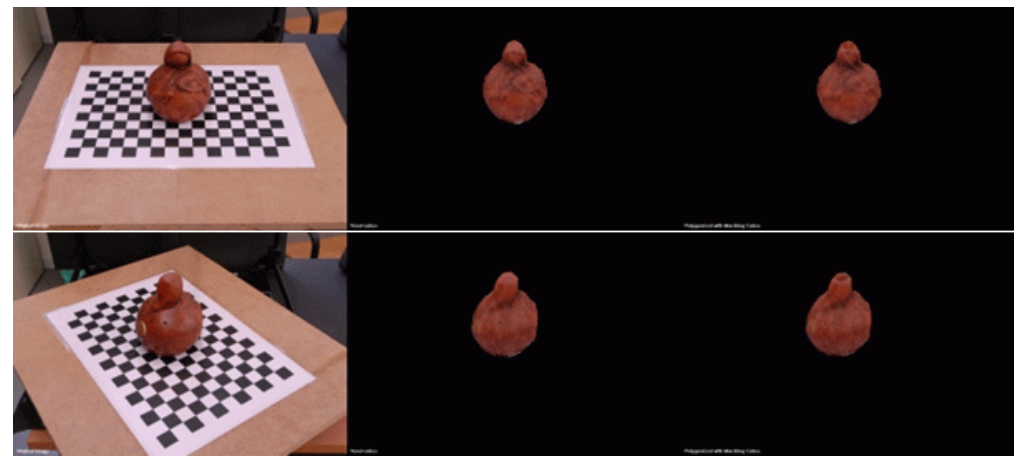

Figure 10: Two different viewpoints (by row) of the 3D reconstruction obtained for the parallelepiped case: on the left, original images; on the centre, voxelized $3 \mathrm{D}$ model and on the right, polygonized 3D model 


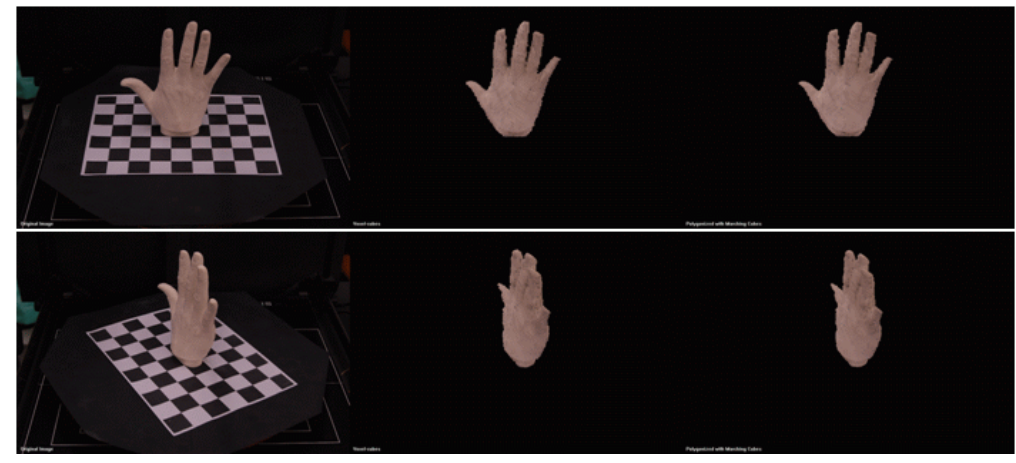

Figure 11: Two different viewpoints (by row) of the 3D reconstruction obtained for the hand model case: on the left, original images; on the centre, voxelized 3D model and on the right, polygonized 3D model
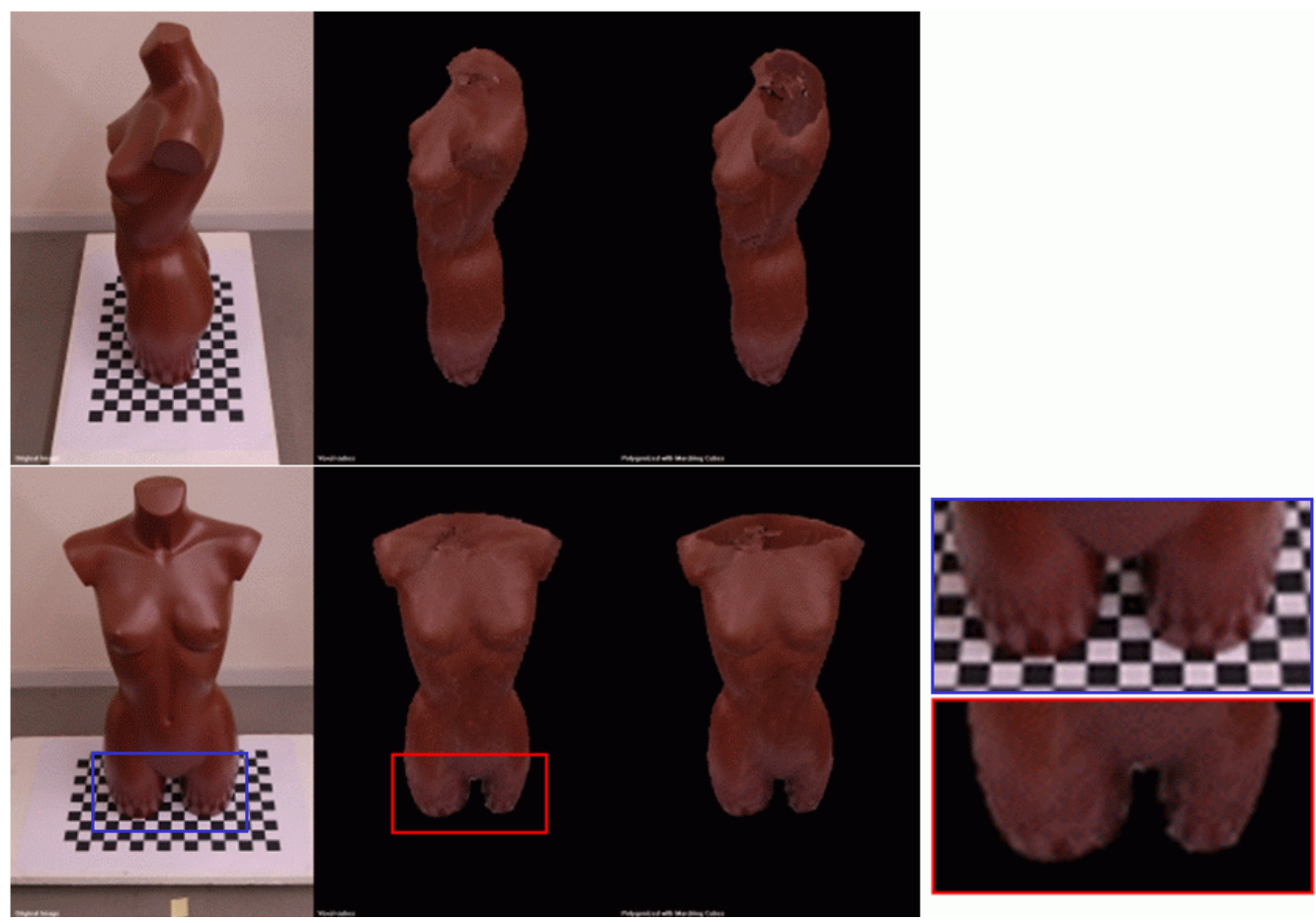

Figure 12: Two different viewpoints (by row) of the 3D reconstruction obtained for the torso model case: on the left, original images; on the centre, voxelized 3D model and on the right, polygonized $3 \mathrm{D}$ model. On the second viewpoint, a close-up view of the lower part of the object and of the reconstructed 3D model

From the voxelized 3D model obtained, some geometrical measures can be determined: height, length and width, for example. Figure 13 resume these values for all reconstructed objects and compares them with the real ones, manually obtained using a simple ruler. 


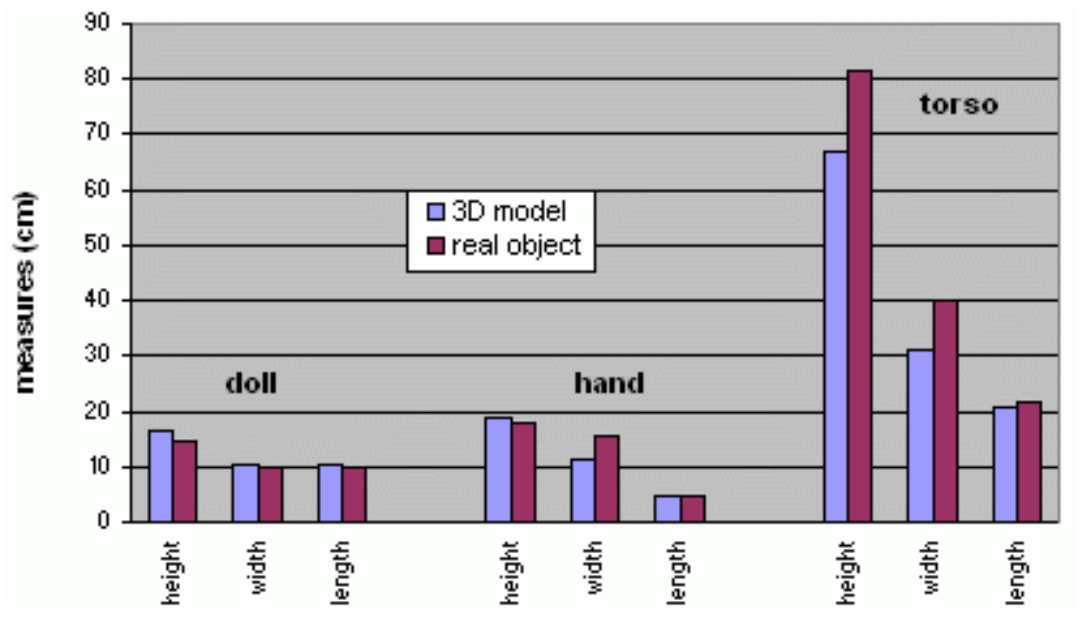

Figure 13: Comparison of some real and measured geometric characteristics obtained from the reconstructed 3D models

The robustness of the GVC method was verified using the hand model object, which has high curvature surfaces, a hard challenge for most reconstruction methods. The test was to reduce the number of images used in the reconstruction process. Initially, 12 images were acquired for the second sequence of all objects. Removing just four images (front, back, right and left side images), the hand model object was again reconstructed. Comparing the obtained 3D model with the previous one (Figure 14), a reduction in its accuracy is evident, especially due to the fingers poor differentiation.
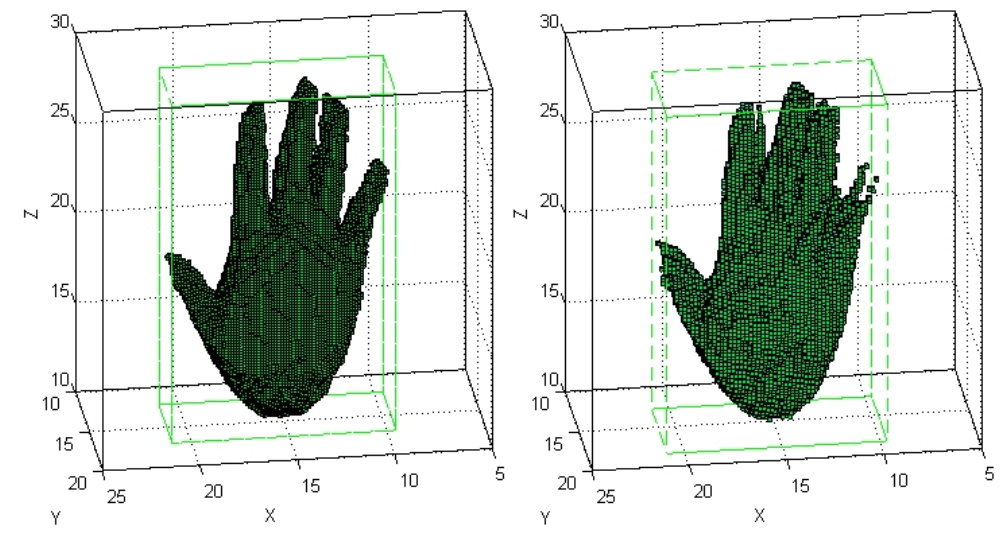

Figure 14: Same viewpoint of the reconstructed (voxelized) 3D models of the hand object.

On the left, the 3D model obtained using all 12 input images; on the right, the 3D

model obtained using just 8 images from the original input image sequence

\section{$5 \quad$ Conclusions and future work}

The quality of the obtained 3D reconstructions is highly dependent on the accuracy of the results of two phases of the methodology used: camera calibration and silhouettes segmentation. Also, the complexity of the objects shape and the reflectance of their surfaces are aspects that must be taken in account for more accurate 3D reconstructions. However, the results obtained were relatively good and suitable for many existing applications, both in terms of shape and appearance. Thus, we can conclude that this methodology is capable of obtaining adequate $3 \mathrm{D}$ reconstructions from images of static objects that can be considered in many nowadays applications. 
Some of our future tasks will be the computational implementation of an auto-calibration method (e.g. [2, $13,38]$ ) and of a methodology for the 3D reconstruction of non-rigid objects (e.g. [42]). Also, some of our attention will be concerning in the implementation of a coarse-to-fine/multi-resolution approach, like it is done in $[29,35]$.

\section{Acknowledgments}

This work was partially done in the scope of the project Segmentation, Tracking and Motion Analysis of Deformable (2D/3D) Objects using Physical Principles, with reference POSC/EEA-SRI/55386/2004, financially supported by FCT - Fundação para a Ciência e a Tecnologia from Portugal.

The first author would like to thank the support of the PhD grant SFRH/BD/27716/2006, also from FCT.

\section{References}

[1] Y. I. Abdel-Aziz and H. M. Karara. Direct linear transformation into object space coordinates in closerange photogrammetry. Symposium on Close-Range Photogrammetry, Urbana, Illinois, IL, USA, 1-18, 1971.

[2] L. d. Agapito, E. Hayman and I. Reid. Self-calibration of a rotating camera with varying intrinsic parameters. British Machine Vision Conference, Southampton, United Kingdom, 105-114, 1998.

[3] M. T. Ahmed, E. E. Hemayed and A. A. Farag. Neurocalibration: A Neural Network That Can Tell Camera Calibration Parameters. International Conference on Computer Vision, Kerkyra, Greece, 463468, 1999.

[4] J. Batista, H. Araújo and A. T. d. Almeida. Iterative Multistep Explicit Camera Calibration. International Conference on Computer Vision, Bombay, India, 15: 709-714, 1998.

[5] J. Čarnický and D. C. Jr. Three-dimensional measurement of human face with structured-light illumination. Measurement Science Review, 6(1): 1-4, 2006.

[6] N. D'Apuzzo. Modeling human faces with multi-image photogrammetry. SPIE Conference on ThreeDimensional Image Capture and Applications, San Jose, CA, USA, 4661: 191-197, 2002.

[7] M. Dooley. Anthropometric modeling programs - a survey. IEEE Computer Graphics and Applications, 2(9): 17-25, 1982.

[8] O. Faugeras. Three-Dimensional Computer Vision: a Geometric Viewpoint. MIT Press, 1993.

[9] D. A. Forsyth and J. Ponce. Computer Vision: A Modern Approach. Prentice Hall Series in Artifical Intelligence, 2003.

[10] J.-S. Franco, M. Lapierre and E. Boyer. Visual Shapes of Silhouette Sets. 3D Processing, Visualization and Transmission, University of North Carolina, Chapel Hill, NC, USA, 2006.

[11] Y. Ge. Noncontact 3D biological shape measurement from multiple views. MSc Thesis, Department of Computer Science, University of Western Australia, Australia, 1994.

[12] J. I. González. Estudio experimental de métodos de calibración y autocalibración de cámaras. $P h D$ Thesis, División de Inteligencia Artificial y Sistemas, Universidad de Las Palmas de Gran Canaria, Spain, 2003.

[13] R. Hartley. Self-Calibration from Multiple Views with a Rotating Camera. European Conference on Computer Vision, Stockholm, Sweden, 1: 471-478, 1994.

[14] R. Hartley and P. Sturm. Triangulation. Computer Vision and Image Understanding, 68(2): 146-157, 1997.

[15] R. Hartley and A. Zisserman. Multiple View Geometry in Computer Vision. Cambridge University Press, 2nd ed., 2004. 
[16] J. Heikkilä and O. Silvén. A Four-step Camera Calibration Procedure with Implicit Image Correction. Computer Vision and Pattern Recognition, San Juan, Puerto Rico, 1106, 1997.

[17] M. Ito. Robot vision modelling - camera modelling and camera calibration. Advanced Robotics, 5(3): 321-335, 1991.

[18] T. Kalkhoven and N. Naber. 3D measurement methods. Research project, Computer-Aided Design and Engineering, Faculty of Industrial Design, University of Technology, Delft, The Netherlands, 2003.

[19] R. Kehl and L. V. Gool. Markerless tracking of complex human motions from multiple views. Computer Vision and Image Understanding, 104(2-3): 190-209, 2006.

[20] L. Kovacs, A. Zimmermann, G. Brockmann, et al. Three-dimensional recording of the human face with a 3D laser scanner. Journal of Plastic, Reconstructive and Aesthetic Surgery, 59(11): 1193-1202, 2006.

[21] K. N. Kutulakos. Shape from the Light Field Boundary. IEEE Computer Vision and Pattern Recognition, San Juan, Puerto Rico, 53-59, 1997.

[22] K. N. Kutulatos and S. M. Steiz. A Theory of Shape by Space Carving. Technical Report TR692, Computer Science Department, University of Rochester, New York, NY, USA, 1998.

[23] A. Laurentini. The visual hull concept for silhouette-based image understanding. IEEE Transactions on Pattern Analysis and Machine Intelligence, 16(2): 150-162, 1994.

[24] M. Loper. Archimedes: Shape Reconstruction from Pictures - A Generalized Voxel Coloring Implementation, 2002, http://matt.loper.org/Archimedes/ (retrieved in August 2006).

[25] W. E. Lorensen and H. E. Cline. Marching cubes: A high resolution 3D surface construction algorithm. International Conference on Computer Graphics and Interactive Techniques, New York, NY, USA, 21(4): 163-169, 1987.

[26] S. J. Marshall and J. H. Gilby. New Opportunities in Non-Contact 3D Measurement. National Measurement Conference, Harrogate, United Kingdom, 2001.

[27] L. Massone, P. Morasso and R. Zaccaria. Shape from occluding contours. SPIE Conference on Intelligent Robots and Computer Vision, Cambridge, MA, USA, 521: 114-120, 1985.

[28] T. McInerney and D. Terzopoulos. Deformable models in medical image analysis: a survey. Medical Image Analysis, 1(2): 91-108, 1996.

[29] A. C. Prock and C. R. Dyer. Towards real-time voxel coloring. DARPA - Image Understanding Workshop, Monterey, CA, USA, 315-321, 1998.

[30] F. Remondino. 3D Reconstruction of Static Human Body with a Digital Camera Videometrics VII, SPIE Conference on Electronic Imaging, California, CA, USA, 5013: 38-45, 2003.

[31] K. Sande. A Practical Setup for Voxel Coloring using off-the-shelf Components. BSc Project, Universiteit van Amsterdam, Netherlands, 2004.

[32] S. Seitz and C. R. Dyer. Photorealistic Scene Reconstruction by Voxel Coloring. Computer Vision and Pattern Recognition Conference, San Juan, Puerto Rico, 1067-1073, 1997.

[33] J. P. Siebert and S. J. Marshall. Human body 3D imaging by speckle texture projection photogrammetry. Sensor Review, 20(3): 218-226, 2000.

[34] G. G. Slabaugh, W. B. Culbertson and T. Malzbender. Generalized Voxel Coloring. Workshop on Vision Algorithms, Corfu, Greece, 100-115, 1999.

[35] G. G. Slabaugh, T. Malzbender and W. B. Culbertson. Volumetric warping for voxel coloring on an infinite domain. Lecture Notes in Computer Science, 2018: 41-50, 2001.

[36] K. Takahashi, T. Sakaguchi and J. Ohya. Remarks on a real-time, noncontact, nonwear, 3D human body posture estimation method. Systems and Computers in Japan, 31(14): 1-10, 2000.

[37] D. Thalmann. Human modelling and animation. Eurographics State of the Art Reports, 1993. 
[38] B. Triggs. Autocalibration and the Absolute Quadric. IEEE Conference on Computer Vision and Pattern Recognition, Puerto Rico, 609-614, 1997.

[39] R. Y. Tsai. A versatile camera calibration technique for high-accuracy 3D machine vision metrology using off-the-shelf tv cameras and lenses. IEEE Journal of Robotics and Automation, 3(4): 323-344, 1987.

[40] F. Tsalakanidou, F. Forster, S. Malassiotis, et al. Real-time acquisition of depth and color images using structured light and its application to 3D face recognition. Real-Time Imaging, special issue on multi-dimensional image processing 11(5-6): 358-369, 2005.

[41] P. Tukuisis, P. Meunier and C. E. Jubenville. Human body surface area: measurement and prediction using three dimensional body scans. European Journal of Applied Physiology and Occupational Physiology, 85(3-4): 264-271, 2001.

[42] S. Vedula, S. Baker, S. Seitz, et al. Shape and motion carving in 6D. IEEE Conference on Computer Vision and Pattern Recognition, Hilton Head Island, SC, USA, 2: 592-598, 2000.

[43] J. Weng, P. Cohen and M. Herniou. Camera calibration with distortion models and accuracy evaluation. IEEE Transactions on Pattern Analysis and Machine Intelligence, 14(10): 965-980, 1992.

[44] K.-Y. K. Wong and R. Cipolla. Structure and Motion from Silhouettes. International Conference on Computer Vision, Vancouver, Canada, 217-222, 2001.

[45] P. Xi, W.-S. Lee and C. Shu. Analysis of segmented human body scans. Graphics Interface, Montreal, Canada, 234: 19-26, 2007.

[46] Y. Yu and J. Malik. Recovering Photometric Properties of Architectural Scenes from Photographs. Computer Graphics, 32: 207-217, 1998.

[47] G. Zeng, M. Lhuillier and L. Quan. Recent Methods for Reconstructing Surfaces from Multiple Images. Lecture Notes in Computer Science, Springer Verlag, 3519: 429-447, 2005.

[48] Z. Zhang. A Flexible New Technique for Camera Calibration. IEEE Transactions on Pattern Analysis and Machine Intelligence, 22(11): 1330-1334, 2000.

[49] L. Zhang, B. Curless and S. M. Seitz. Rapid Shape Acquisition Using Color Structured Light and Multi-pass Dynamic Programming. 1st International Symposium on 3D Data Processing, Visualization, and Transmission, Padova, Italy, 24-36, 2002. 